\title{
Early treatment of obstructive apnoea and stroke outcome: a randomised
} controlled trial

\author{
O. Parra, Á. Sánchez-Armengol, M. Bonnin, A. Arboix, F. Campos-Rodríguez, \\ J. Pérez-Ronchel, J. Durán-Cantolla, G. de la Torre, J.R. González Marcos, \\ M. de la Peña, M. Carmen Jiménez, F. Masa, I. Casado, M. Luz Alonso and J.L. Macarrón
}

ABSTRACT: The aim of the present study was to assess the impact of nasal continuous positive airway pressure (nCPAP) in ischaemic stroke patients followed for 2 yrs.

Stroke patients with an apnoea-hypopnoea index $\geqslant 20$ events $\cdot \mathrm{h}^{-1}$ were randomised to early nCPAP ( $n=71$; 3-6 days after stroke onset) or conventional treatment $(n=69)$. The Barthel Index, Canadian Scale, Rankin Scale and Short Form-36 were measured at baseline, and at 1, 3, 12 and 24 months.

The percentage of patients with neurological improvement 1 month after stroke was significantly higher in the nCPAP group (Rankin scale 90.9 versus $56.3 \%(p<0.01)$; Canadian scale 88.2 versus $72.7 \%(p<0.05)$ ). The mean time until the appearance of cardiovascular events was longer in the nCPAP group (14.9 versus 7.9 months; $p=0.044$ ), although cardiovascular event-free survival after $\mathbf{2 4}$ months was similar in both groups. The cardiovascular mortality rate was $0 \%$ in the nCPAP group and $4.3 \%$ in the control group $(p=0.161)$.

Early use of nCPAP seems to accelerate neurological recovery and to delay the appearance of cardiovascular events, although an improvement in patients' survival or quality of life was not shown.

KEYWORDS: Cardiovascular events, ischaemic stroke, mortality, nasal continuous positive airway pressure, neurologic outcome, quality of life

leep-related breathing disorders are associated with an increase in cardiovascular morbidity and mortality in general [1-3], and sleep-disordered breathing occurs frequently in stroke patients [4-6]. It has been shown that obstructive sleep apnoea (OSA) is a risk factor for stroke $[7,8]$, and an independent predictor of outcome in terms of functional recovery [9] and mortality [10, 11]. Because there are data in favour of the physiopathological plausibility of this association [12-15], it seems reasonable to perform intervention studies of treatment with continuous positive airway pressure (CPAP) in stroke patients. Nasal CPAP (nCPAP) is the most effective treatment for OSA [16, 17] and for OSAassociated hypertension [18-21], which has been suggested to act on the underlying mechanisms by which OSA increases the risk of stroke [22-24].

Because stroke and sleep-related breathing disorders are frequent and interrelated conditions, with a deleterious impact in older people [25], the possibility of demonstrating a favourable effect of treatment with nCPAP could be great of social and economic importance. In fact, clinical data available to date regarding the use of nCPAP in the management of stroke patients with OSA are still scarce and controversial [26-28], with both encouraging [29] and disappointing [30-33] results. In a recent open-label 5-yr follow-up study of stroke patients with moderate-severe sleep apnoea, nCPAP treatment started in the stable phase of the disease was associated with a reduction in the excess risk of mortality found in OSA patients with stroke [34].

To shed light on literature discrepancies, we explored the robustness of previous findings in patients with first-ever ischaemic stroke and OSA using a randomised controlled design. The objectives of the study were: 1) to assess the benefits of 2 yrs of early nCPAP treatment on

For editorial comments see page 997.

Earn CME accreditation by answering questions about this article. You will find these at the back of the printed copy of this issue or online at www.erj.ersjournals.com/misc/cmeinfo.xhtml
AFFILIATIONS

A full list of the authors' affiliations can be found in the Acknowledgements section.

CORRESPONDENCE

0. Parra

Dept of Pneumology

Hospital Universitari del Sagrat Cor C/Viladomat 288

E-08029 Barcelona

Spain

E-mail: oparra@ub.edu

Received:

March 042010

Accepted after revision:

Aug 012010

First published online:

Sept 162010 
functional outcome, quality of life, appearance of new cardiovascular events and mortality; and 2) to assess the feasibility of using nCPAP in the early phase of stroke.

\section{PATIENTS AND METHODS}

\section{Design overview}

A prospective, randomised, controlled and multicentre study was designed to test the hypothesis that early nCPAP treatment may favourably affect the outcome of patients with first-ever ischaemic stroke, in terms of neurologic improvement, quality of life, occurrence of new cardiovascular events and mortality.

\section{Setting and participants}

Between September 2005 and December 2006, all patients with first-ever ischaemic stroke admitted consecutively to the neurology services of seven acute-care teaching hospitals throughout Spain were eligible. Inclusion criteria were age $<75$ yrs and at least one of the following conditions: habitual snoring, observed apnoeas, or history of hypertension or ischaemic heart disease. Patients with consciousness impairment and patients previously diagnosed and treated for OSA were excluded. The protocol consisted of complete neurological assessment, cardiovascular risk factors, health-related quality of life and sleep studies. Patients with moderate-severe sleep apnoea, defined as an apnoea-hypopnoea index (AHI) $\geqslant 20$ events $\cdot h^{-1}$, were randomised to receive conventional treatment (described later) for stroke plus nCPAP (nCPAP group) or conventional treatment without nCPAP (control group) during the acute phase of stroke, and were followed for 24 months.

The study was approved by institutional review boards of the participating centres and written informed consent was obtained from all patients or their families.

\section{Study procedures}

Neurological and outcome data were recorded following the standardised protocol of the Hospital del Sagrat Cor Stroke Registry [35]. Stroke subtypes were classified according to the Cerebrovascular Study Group of the Spanish Society of Neurology [36] and include transient ischaemic attack, ischaemic stroke (either atherothrombotic, cardioembolic, lacunar, unusual or of undetermined origin) and intraparenchymatous haemorrhagic stroke. For the purpose of this study, patients with ischaemic stroke were selected.

Functional abilities were assessed using the Barthel index [37], a multifaceted scale questionnaire that measures morbidity and daily living activities (score ranges from 0 (maximal disability) to 100 (no disability)), where a score $<20$ indicates that the person is totally dependent, a score between 20 and 35 a severe level of disability, a score between 40 and 55 a moderate level of disability, a score $\geqslant 60$ a mild level of disability and 100 no disability or that the person is totally independent. The maximal severity of stroke or neurological impairment was estimated using the Canadian scale [38] (total score ranges from 0 (maximal impairment) to 10 (no impairment)). The modified Rankin scale [39] was used to assess outcome (scores ranges from 0 (no symptoms) to 6 (death)). Self-reported health status was assessed with the Short Form
(SF)-36 quality of life questionnaire [40]; the physical (PCS) and mental (MCS) component summaries were calculated.

\section{Sleep studies}

A sleep-wake habits and symptoms questionnaire that consisted of 15 items, including snoring, observed apnoea and hypersomnia in different situations, was applied within the first $48-72 \mathrm{~h}$. The possible answers were never, rarely, sometimes, often and always. Details of the questionnaire have been reported previously [6]. Daytime sleepiness was also assessed with the Epworth Sleepiness Scale [41]. Answers were obtained from the patients themselves or with the aid of relatives, if needed.

A respiratory sleep study was performed in the ward during the first $48-72 \mathrm{~h}$ after admission with a portable respiratory recording device (Hypno TT Digital Recorder; Tyco/ Healthcare/Puritan Bennett, Villers-lès-Nancy, France) that have been previously validated using full polysomnography and used in stroke patients [6]. Respiratory nasal airflow (nasal flow sensor), chest wall movements (impedance), heart rate and thoracic impedance (ECG electrodes), arterial oxygen saturation measured by pulse oximetry $\left(\mathrm{Sp}_{\mathrm{p}} \mathrm{O}_{2}\right.$; finger pulse oximeter) and body position (position sensor) were measured. Sleep-related breathing disorders were classified as obstructive or central apnoea, with apnoea considered a cessation of airflow for $\geqslant 10 \mathrm{~s}$ with maintenance of thoracic motion or without any thoracic motion, respectively. A hypopnoea was considered a discernible reduction in airflow or thoracic motion which lasted $>10 \mathrm{~s}$ and was associated with a cyclical dip in $\mathrm{Sp}_{\mathrm{O}} \mathrm{O}_{2}$ of $>3 \%$. The $\mathrm{AHI}$ was calculated taking into account the time spent in bed with the respiratory recording device. In all cases, scoring of these variables was performed manually by an experienced scorer. The percentage of the night with $\mathrm{Sp}_{\mathrm{p}, \mathrm{O}_{2}}<90 \%$ (CT90) was obtained automatically.

\section{Randomisation and intervention}

Patients with an AHI $\geqslant 20$ events $\cdot h^{-1}$ (with predominantly obstructive events) were randomised to receive conventional treatment for stroke plus nCPAP or conventional treatment without nCPAP (control group) using a computer-generated random list (1:1 ratio). Autotitration polygraphic studies were carried out using a validated portable system (Autoset Portable Plus II; ResMed, Sydney, Australia) [42]. The optimal pressure was determined visually on the raw data of the auto-CPAP device ("view night profile") by analysing the pressure that included $90 \%$ of the periods with a leak $<0.4 \mathrm{~L} \cdot \mathrm{s}^{-1}$ (90th percentile), providing the pressure that normalises respiratory disturbances index [43]. Therefore, a fixed nCPAP prescribed was derived from examination of the profiles of the pressure applied during autotitration CPAP.

In all patients assigned to the intervention arm, nCPAP was started during hospital admission between the first 3 and 6 days after the onset of stroke. In all cases, nCPAP was administered by well-trained nurses of the sleep units of the participating hospitals

In the acute phase of stroke, patients were managed in accordance with recommendations of the Spanish Cerebrovascular Study Group of the Spanish Society of Neurology, as previously described [36]. The main strategies were as follows: 
1) maintenance of blood pressure without the use of hypotensive drugs unless the systolic blood pressure was $\geqslant 220$ $\mathrm{mmHg}$ or the diastolic blood pressure $\geqslant 120 \mathrm{mmHg}$; 2) early treatment of hyperglycaemia avoiding the use of glucose infusion; 3) prevention of pulmonary thromboembolism with low-dose heparin; and 4) early antiplatelet therapy, except when anticoagulation was recommended. Physical and respiratory therapy was performed during the patient's stay in the hospital.

\section{Outcomes and follow-up}

After hospital discharge, patients were followed by a neurologist and a pneumologist at the outpatient clinics at 1, 3, 12 and 24 months after stroke. In all visits, a physical examination was performed, and the Barthel index, Canadian scale, Rankin scale and SF-36 were administered. nCPAP treatment was considered adequate when the system counter registered $>4 \mathrm{~h}(70 \%$ of the days) per night, checked during all medical visits undertaken during the study. Cardiovascular events including cardiac ischaemic events, stroke recurrence and cardiovascular mortality were recorded during the study visits, and asked by phone at the end of the study for every patient.

\section{Statistical analysis}

A per-protocol analysis was carried out, so that patients who refused nCPAP during hospitalisation were excluded. A descriptive study of baseline characteristics of the study sample was performed. To check the homogeneity of the study groups, we performed between-group comparisons (OSA group with nCPAP versus OSA group without nCPAP) with regard to demographic data, sleep-related data, respiratory polysomnography and neurological data, using one-factor ANOVA and the Pearson's Chi-squared tests. Mean scores of the Barthel index, Canadian Scale, Rankin scale and SF-36 at 1, 3, 12 and 24 months of follow-up were compared with baseline data (except for SF-36, in which the last three visits were compared with visit at 1 month) were compared with t-tests for paired samples, and between groups with one-factor ANOVA. Improvement variables of neurological scales included the following: improvement of the Barthel index (improvement in the level of disability or no disability from the beginning), improvement of the Rankin scale (reduction of $\geqslant 1$ point or score 0 from the beginning) and improvement in the Canadian scale (increases of $\geqslant 5$ points or score 10 from the beginning). The percentage of patients that showed an improvement in the neurological scales at 1 month after stroke in both groups was compared with the Chi-squared test and odds ratio calculation.

To study the incidence of exitus (overall mortality), cardiovascular death (cardiovascular mortality) and the incidence of cardiovascular events (including cardiac ischaemic events, stroke recurrence and cardiovascular death), we used univariate tests for the comparison of proportions (Chi-squared tests and calculation of odds ratios). Moreover, the time elapsed from the onset of stroke to the appearance of cardiovascular events in both groups was compared with the Kolmogorov-Smirnov Z-test.

Direct survival analysis of the cardiovascular events, including stroke recurrence and/or cardiovascular exitus, were also performed, using Kaplan-Meier tests.
To evaluate the related factors we performed univariate tests (Chi-squared tests and calculation of odds ratios) and multivariable analyses with Cox proportional-hazards regression models, which included, in addition to the study group (nCPAP or control group), the AHI, CT90, factors such as sex, age, body mass index (BMI), history of hypertension, ischaemic heart disease, smoking, and scores on the Barthel, Canadian, ESS and Rankin scales. Significance was set at $p<0.05$. SPSS (version 13.0 for Windows; IBM, Madrid, Spain) was used for statistical analysis.

\section{RESULTS}

Of a total of 235 patients with first-ever ischaemic stroke recruited during the study period and undergoing a respiratory sleep study during the acute phase of stroke, 140 met the criteria of $\mathrm{AHI} \geqslant 20$ events $\cdot \mathrm{h}^{-1}$ and were randomised to the nCPAP $(n=71)$ or control groups $(n=69)$. However, $20(28.2 \%)$ out of the 71 patients in which nCPAP treatment was started refused nCPAP due to machine discomfort (14 patients were excluded because of nCPAP refusal after one to three nights of treatment, and the remaining six patients not excluded during their hospital stay refused nCPAP at follow-up after a mean of 10 months of treatment). Therefore, the study population consisted of 57 patients assigned to the nCPAP group and 69 to the control group (fig. 1). Treatment with nCPAP was started at a mean \pm SD of $4.6 \pm 2.8$ days after the onset of stroke.

Patients in both study groups showed similar baseline characteristics (table 1). The groups were balanced in terms of drugs administered in the hospital acute phase. The baseline characteristics of the 14 patients excluded from the study because of refusal of nCPAP during hospitalisation were similar to those of the whole study population. The mean $\pm \mathrm{SD}$ length of hospital stay was $8.7 \pm 4.3$ days in the nCPAP group and $9.3 \pm 4.6$ days in the control group, without statistically significant differences. There were no in-hospital deaths and all patients were discharged alive from the hospital.

Patients were followed for a mean \pm SD of $23.04 \pm 3.7$ months. The mean \pm SD nCPAP use was $5.3 \pm 1.9 \mathrm{~h} \cdot$ night $^{-1}$ during a mean of $6.8 \pm 0.6$ nights week $^{-1}$. The mean nCPAP pressure was $8.6 \pm 1.5 \mathrm{cmH}_{2} \mathrm{O}$. A face mask was necessary in only four patients because of leaks, mainly due to facial palsy.

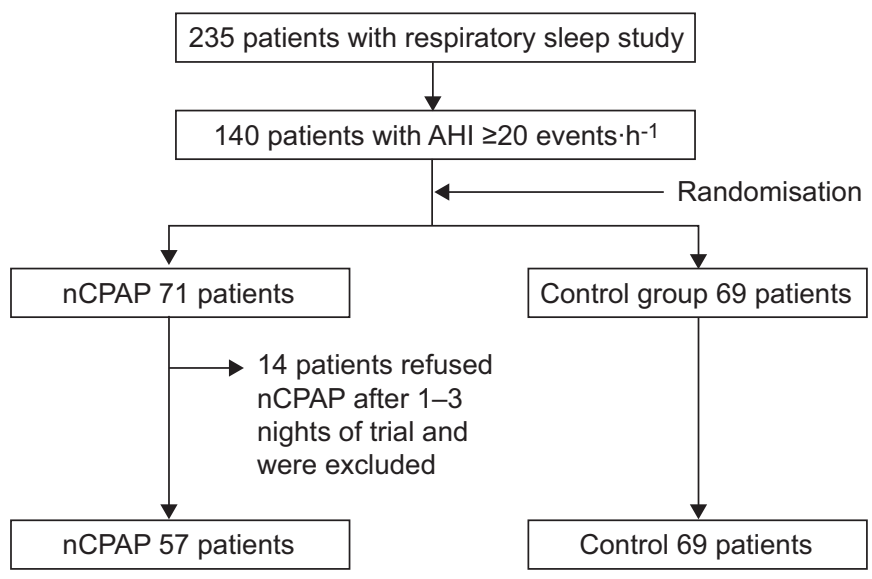

FIGURE 1. Flow chart of the study population. AHI: apnoea-hypopnoea index nCPAP: nasal continous positive airway pressure. 
TABLE 1 Clinical characteristics of 126 patients with ischaemic stroke and moderate-severe obstructive sleep apnoea, according to randomisation to the study groups

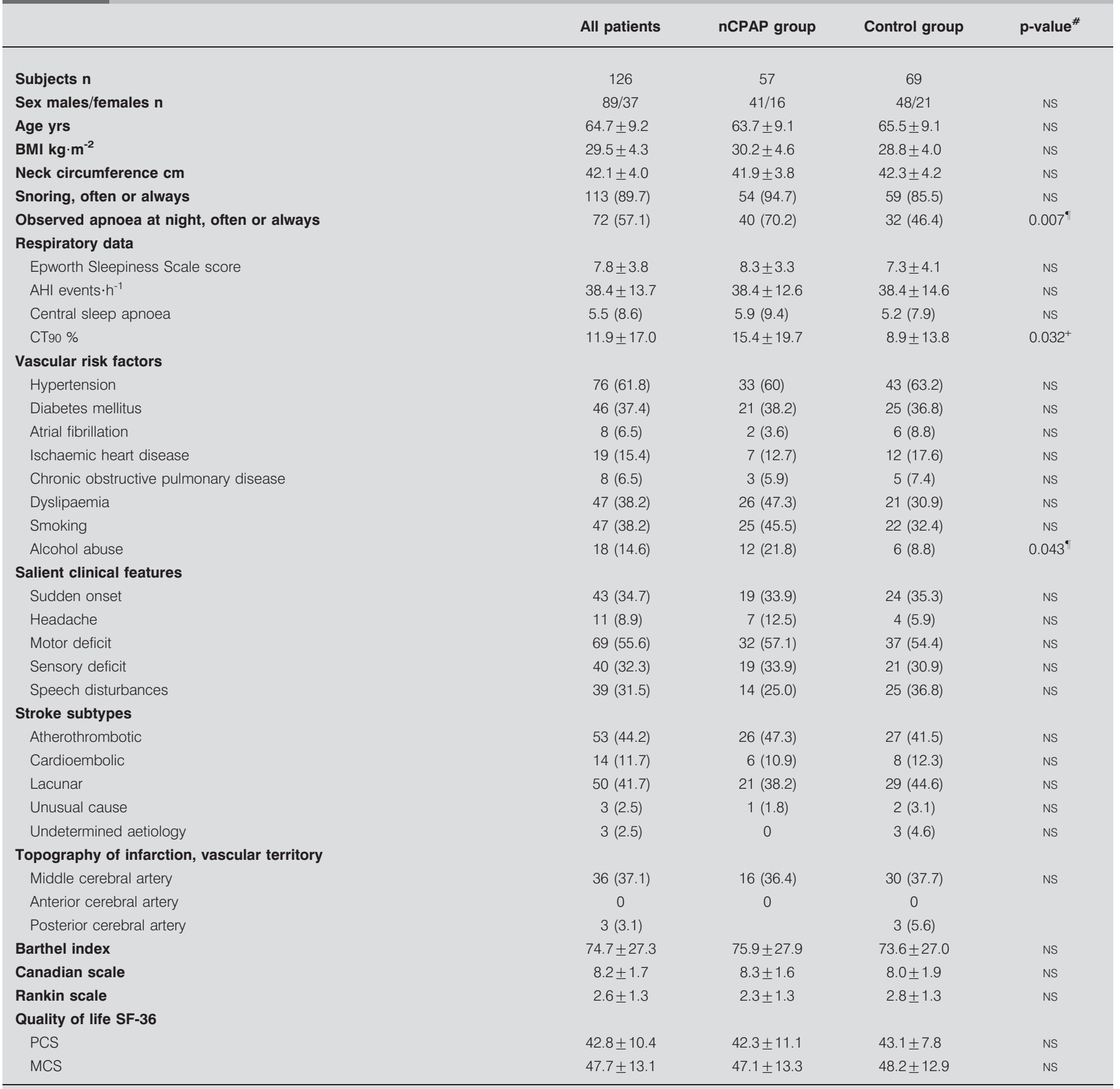

Data as presented as mean \pm SD or $n(\%)$, unless otherwise stated. $\mathrm{nCPAP}$ : nasal continuous positive airway pressure; BMI: body mass index; CT90: percentage of the night with arterial oxygen measured by pulse oximetry <90\%; SF: Short Form; PCS: physical component summary; MCS: mental component summary; NS: nonsignificant. ${ }^{\#}$ : reflect differences between the nCPAP group and the control group; ": Chi-squared test; ${ }^{+}$: one-factor ANOVA.

In both groups, there were significant changes in the Barthel, Canadian and Rankin scales in all visits as compared with baseline (fig. 2). The percentage of patients with improvements in the neurological assessments 1 month after stroke was significantly higher in the nCPAP group than in controls in the Rankin scale (90.9 versus $56.3 \%$; OR $7.8 ; \mathrm{p}<0.01$ ) and the
Canadian scale ( 88.2 versus $72.7 \%$; OR $2.8 ; \mathrm{p}<0.05$ ), even when patients with less severe neurological impairment (baseline score in the Rankin scale 1 and baseline score in the Canadian scale 10) were excluded. However, significant differences in the percentage of patients with improvements in the Barthel index were not observed (table 2). Although almost all mean 

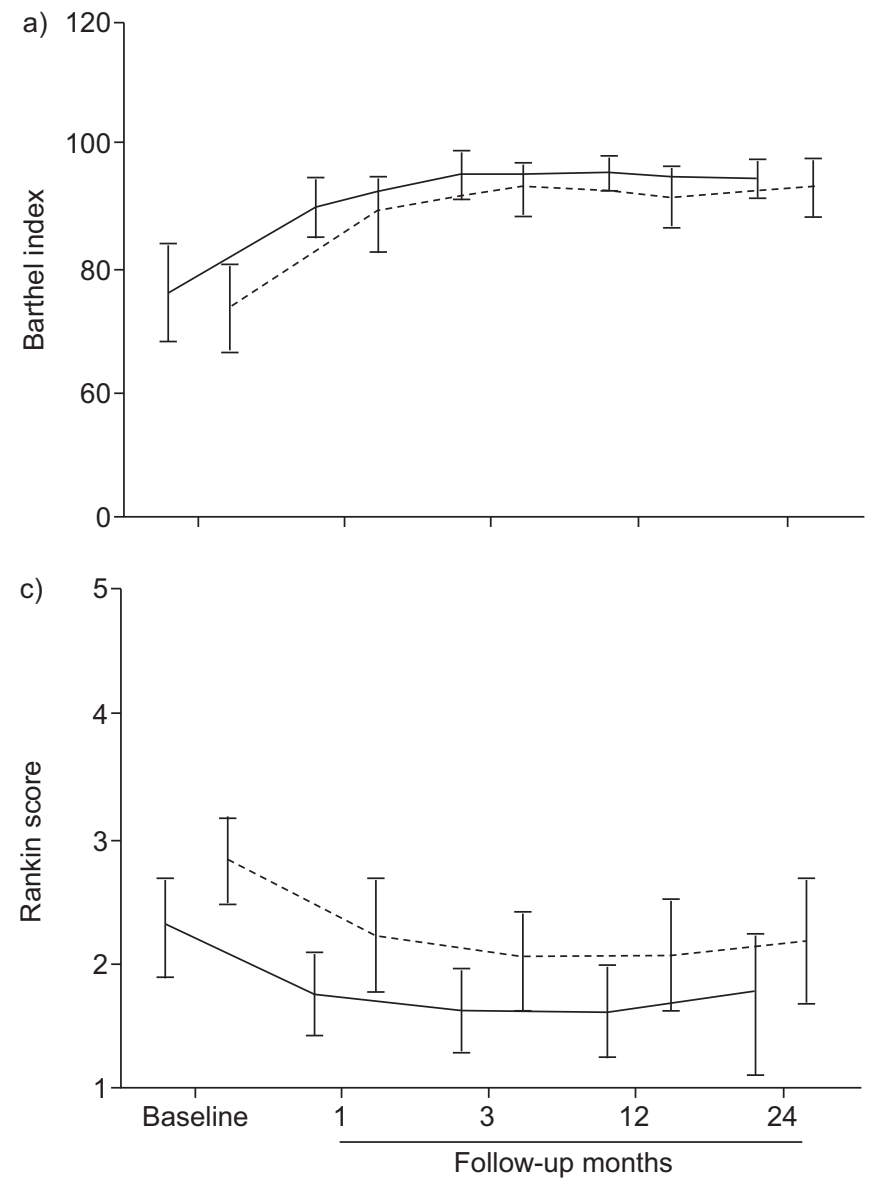

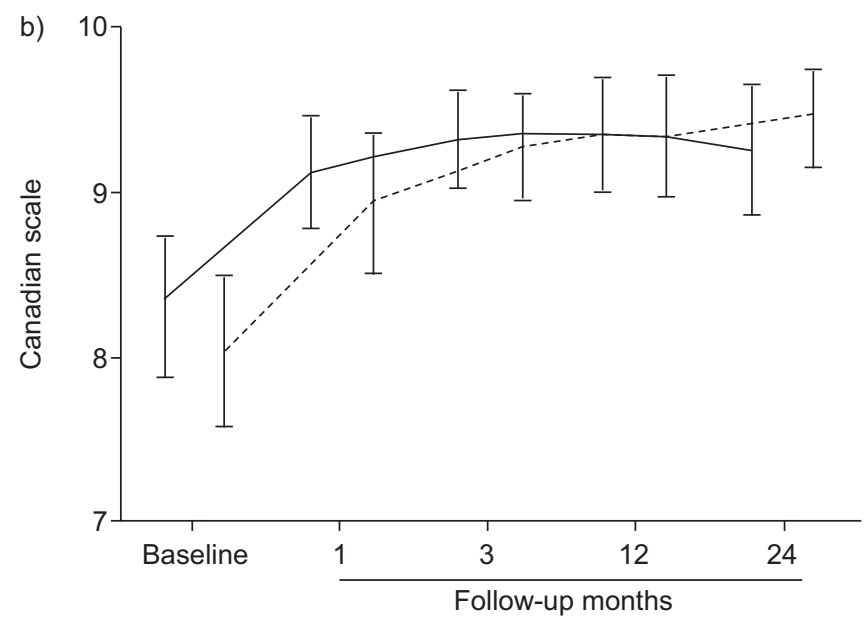

FIGURE 2. Changes in the mean values of the a) Barthel index, b) Canadian scale and c) Rankin score throughout the study. ----: control group; - — : nasal continuous positive airway pressure group.

scores of the neurological scales, and the PCS and MCS of the SF-36 were better in the nCPAP group in all follow-up visits, statistically significant differences were not observed (table 3).

Outcome in terms of mortality and new cardiovascular events (including stroke recurrence and cardiovascular deaths) is shown in table 4 . The cardiovascular mortality rate was $0 \%$ in the nCPAP group $(n=0)$ compared with $4.3 \%$ in the control group $(n=3)(p=0.161)$. The rate of cardiovascular events (including cardiac ischaemic events, stroke recurrence and cardiovascular death) was $12.3 \%$ (seven out of 57 subjects) in the nCPAP group and $11.6 \%$ (eight out of 69 subjects) in the

\section{TABLE 2 Percentage of patients with improvement in neurological parameters 1 month after stroke}

nCPAP group

\section{Subjects $n$}

Barthel index, improvement $\geqslant 1$ point of disability

All patients

Excluding patients with less severe stroke

Rankin scale, reduction $\geqslant 1$ point per category

All patients

Excluding patients with less severe stroke

Canadian scale, increase of $\geqslant 0.5$ points

All patients

Excluding patients with less severe stroke

\section{Control group}

69

$43 / 52(82.7)$

26/35 (74.3)

45/54 (83.3)

$30 / 39(76.9)$

0.567

0.502

$30 / 33(90.9)$

$21 / 24(87.5)$

$18 / 32(56.3)$

$14 / 28(50.0)$

$45 / 51(88.2)$

$33 / 39(84.6)$

OR (95\% Cl)

p-value
Data are presented as $\mathrm{n} / \mathrm{N}(\%)$, unless otherwise stated. nCPAP: nasal continuous positive airway pressure. ${ }^{*}$ : between the nCPAP group and the control group (Chi-squared test). 


\begin{tabular}{|c|c|c|c|}
\hline \multirow[t]{2}{*}{ TABLE 3} & \multicolumn{3}{|c|}{$\begin{array}{l}\text { Follow-up data in the groups with and without } \\
\text { nasal continuous positive airway pressure } \\
\text { (nCPAP) }\end{array}$} \\
\hline & nCPAP group & Control group & p-value ${ }^{\#}$ \\
\hline Subjects n & 57 & 69 & \\
\hline \multicolumn{4}{|l|}{ Barthel index } \\
\hline Baseline & $75.9 \pm 27.9$ & $73.6 \pm 27.0$ & NS \\
\hline 3 months & $95.0 \pm 13.4$ & $92.8 \pm 17.8$ & NS \\
\hline 12 months & $95.3 \pm 10.0$ & $91.4 \pm 17.8$ & NS \\
\hline 24 months & $94.3 \pm 10.9$ & $93.1 \pm 15.8$ & NS \\
\hline \multicolumn{4}{|l|}{ Canadian scale } \\
\hline Baseline & $8.3 \pm 1.6$ & $8.0 \pm 1.9$ & NS \\
\hline 3 months & $9.3 \pm 1.0$ & $9.3 \pm 1.3$ & NS \\
\hline 12 months & $9.4 \pm 1.2$ & $9.4 \pm 1.3$ & NS \\
\hline 24 months & $9.3 \pm 1.3$ & $9.5 \pm 1.0$ & NS \\
\hline \multicolumn{4}{|l|}{ Rankin scale } \\
\hline Baseline & $2.3 \pm 1.3$ & $2.8 \pm 1.3$ & NS \\
\hline 3 months & $1.6 \pm 0.9$ & $2.0 \pm 1.1$ & NS \\
\hline 12 months & $1.6 \pm 0.9$ & $2.1 \pm 1.2$ & NS \\
\hline 24 months & $1.8 \pm 1.1$ & $2.2 \pm 1.1$ & NS \\
\hline \multicolumn{4}{|l|}{ SF-36 } \\
\hline \multicolumn{4}{|l|}{ PCS } \\
\hline 1 month & $42.6 \pm 10.2$ & $42.3 \pm 11.8$ & NS \\
\hline 3 months & $44.9 \pm 9.2$ & $44.8 \pm 11.8$ & NS \\
\hline 12 months & $46.7 \pm 8.8$ & $46.5 \pm 11.7$ & NS \\
\hline 24 months & $45.8 \pm 10.0$ & $46.0 \pm 9.8$ & NS \\
\hline \multicolumn{4}{|l|}{ MCS } \\
\hline 1 month & $43.3 \pm 13.2$ & $43.7 \pm 14.1$ & NS \\
\hline 3 months & $46.9 \pm 10.9$ & $46.3 \pm 14.4$ & NS \\
\hline 12 months & $49.1 \pm 14.0$ & $44.6 \pm 12.8$ & NS \\
\hline 24 months & $47.6 \pm 13.8$ & $47.8 \pm 12.1$ & NS \\
\hline
\end{tabular}

Data are presented as mean $\pm \mathrm{SD}$, unless otherwise stated. SF: Short Form; PCS: physical component summary; MCS: mental component summary; NS: nonsignificant. ${ }^{\#}$ : between the nCPAP group and the control group (one-factor ANOVA).

control group $(p=0.560)$. The mean time from stroke onset until the appearance of the first cardiovascular event was significantly longer in the nCPAP group than in the control group (14.9 versus 7.9 months; $\mathrm{p}=0.044)$. The overall cardiovascular eventfree survival rate after 24 months was $87.7 \%$ (50 out of 57 subjects) in the nCPAP group and $88.4 \%$ (61 out of 69 subjects) in the control group (log-rank test $0.01 ; \mathrm{p}=0.911$; fig. 3 ).

In the univariate and multivariable analysis of factors associated with cardiovascular events or mortality at followup, none of the variables analysed, including sex, age, BMI, snoring, observed apnoea, history of hypertension, ischaemic heart disease, smoking, dyslipidaemia, Epworth Sleepiness scale, Barthel index, Canadian scale, Rankin scale, AHI, CT90 or $\mathrm{nCPAP}$, were associated with a higher risk (variable $\mathrm{nCPAP}$ hazard ratio $0.62,95 \%$ CI 3.46-0.11; $\mathrm{p}=0.586$ ).

\section{DISCUSSION}

The present results indicate that early use of nCPAP in patients with a first-ever ischaemic stroke and moderate-severe OSA is associated with a significant improvement in the assessment of neurological scales at 1 month after stroke in the nCPAP group

\begin{tabular}{|c|c|c|}
\hline & nCPAP group & Control group \\
\hline Subjects & 57 & 69 \\
\hline \multicolumn{3}{|l|}{ Cardiovascular events } \\
\hline Stroke & 3 & 3 \\
\hline Transient ischaemic attack & 1 & \\
\hline Angina & 1 & \\
\hline Myocardial infarction & 1 & \\
\hline Other events & 1 & 2 \\
\hline Deaths & 2 & 3 \\
\hline Cardiovascular-related deaths & & 3 \\
\hline Noncardiovascular-related deaths & 2 & \\
\hline
\end{tabular}

compared with controls, whereas in the subsequent follow-up visits, although more favorable results were obtained in the nCPAP group, differences were not significant, probably because patients in both groups had already recovered from the acute phase of stroke. Acceptance of nCPAP therapy and adherence to treatment were highly acceptable, despite the fact that nCPAP was started early in the acute phase of stroke. After a follow-up of 24 months, a significant increase in the length of time until the appearance of cardiovascular events (almost double) was seen in the nCPAP group, as well as a low cardiovascular mortality (no case of cardiovascular death versus three deaths in the control group), although without statistically significant differences, and a cardiovascular eventfree survival similar to that in control patients. To our knowledge, this is the first study of early nCPAP carried out in a homogeneous group of patients with acute first-ever ischaemic stroke. Patients with recurrent stroke would present a more severe clinical condition, presumably due to previous neurological deficits, which would affect the follow-up of these cases, and for these reasons, patients with previous stroke were

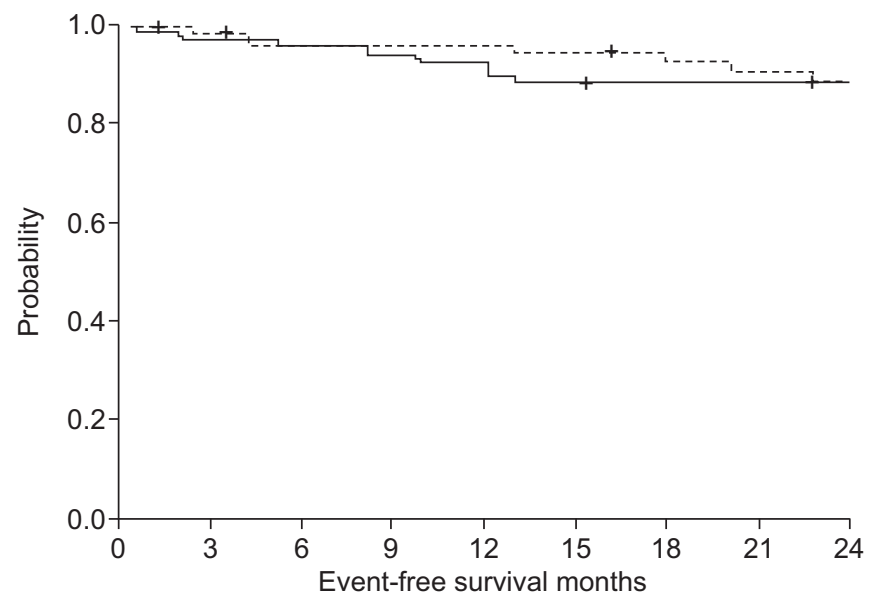

FIGURE 3. Overall cardiovascular event-free survival in the nasal continuous positive airway pressure (nCPAP) and control groups. -----: with nCPAP (50 (87.72\%) out of 57 subjects); — - without nCPAP (61 (88.41\%) out of 69 subjects). Log rank=0.01; $p=0.911$. 
excluded. However, randomised controlled studies of nCPAP therapy in acute stroke patients introduced within the first days after stroke onset and assessing quality of life, in addition to neurological outcomes, recurrence of cardiovascular events and mortalitys have not previously been reported.

Different studies have analysed the influence of sleep-related breathing disorders as cardiovascular [1-3] and cerebrovascular $[7,8]$ risk factors, as well as the prognostic implications of OSA [9-11]. In the present study, we choose AHI $\geqslant 20$ events $\cdot h^{-1}$, because an excess of mortality has been previously described in elderly people [44] and because using respiratory polygraphy, the AHI can be underestimated. However, a few studies have systematically assessed the effect of nCPAP therapy. Studies with more promising results [26-29] are noncontrolled observational evaluations of clinical series of patients, with adherence rates $<50 \%$, poorly defined outcomes, assessments carried out at different time-points during the clinical course of stroke, and lack of medium- or long-term follow-up data. However, less encouraging studies have been reported either by the absence of significant differences between nCPAP and non-nCPAP groups in a small number of patients or by the total inability of using nCPAP in the acute phase of stroke [30-33]. Reviews of OSA and the risk of stroke have repeatedly claimed the need for randomised controlled trials [45].

It is generally believed that nCPAP treatment in stroke patients is difficult, particularly in the acute phase of stroke during inhospital care. Although it may be argued that in our study, severe stroke patients were probably excluded because only first-ever stroke patients with an unaltered level of consciousness were eligible, encouragement and full explanation of the usefulness of nCPAP were positive drivers to obtain noncompliance rates at follow-up that were similar to those reported in patients with OSA syndrome [46]. However, in order to facilitate adherence to nCPAP, age $<75$ yrs was an inclusion criterion, which probably accounted for the exclusion of stroke patients with a more severe condition, as shown by the low mortality rate observed in this cohort. Although one concern is choosing the moment for starting nCPAP treatment given neurological deficits, including facial palsy, and impaired psychological status of stroke patients immediately after stroke, early use of nCPAP may prevent recurrent hypoxaemia and cerebral flow fluctuations due to apnoea that could damage the area of ischaemic penumbra and, therefore, affect prognosis [47]. In this case, nCPAP could exert a beneficial influence in the acute phase. The lack of somnolence in these patients in another factor that may account for poor adherence. The fact that placebo nCPAP was not used is a limitation of the study. A per-protocol analysis was carried out and for this reason, the 14 patients who refused nCPAP during hospitalisation were excluded. Although and intention-to-treat analysis would perhaps be a more rigorous approach, the plausibility that including such patients in the nCPAP group would improve nCPAP results is really poor. However, the other six patients who had poor tolerance to nCPAP, with a mean use of 10 months, were maintained in the group, but no differences were observed when data were reanalysed excluding these patients.

The present findings have shown a favorable effect of nCPAP on the short-term neurological recovery (1 month) but no significant differences were found in the subsequent follow-up visits, although a constant trend in almost all parameters analysed to obtain a higher improvement in patients with nCPAP was observed. It may be hypothesised that differences may be more evident with a larger sample size, but it seems that benefits of nCPAP may appear in stroke patients with a more severe condition, rather than with a higher number of cases. It was found that excluding patients with less severe neurological impairment, results at 1 month were consistently significant despite a reduction in the number of patients.

Another possibility is that the parameters used to assess the patients' outcomes, which were mainly clinical, may not be sufficiently sensitive. The stroke measures used in this study have been criticised for the low sensitivity in patients with minor stroke. In the case of the Barthel scale, a "ceiling effect" of $64.6 \%$ in patients with minor stroke and of $24.8 \%$ in patients with moderate stroke has been reported [48], which may probably account for the inconclusive results in the Barthel index obtained in this study.

It is possible that the evaluation of new silent ischaemic lesions may provide positive results in favour of the nCPAP therapy, but unfortunately this comparison could not be made because magnetic resonance imaging studies at the time of diagnosis were not available in all patients [24, 49]. In this respect, it would be also interesting to assess the information provided by neuropsychological performance studies $[50,51]$ and voxelbased morphometry analysis [52].

In relation to cardiovascular deaths, the trend found in our study was towards a lower mortality in the nCPAP group, but early nCPAP treatment in ischaemic stroke patients had no effect to reduce the occurrence of new cardiovascular events, including ischaemic cardiac events, stroke recurrences or mortality. These findings are, in some ways, consistent with data recently reported by MARTINEZ-GARCIA et al. [34] in a 5-yr follow-up study, in which 68 ischaemic stroke patients with an $\mathrm{AHI} \geqslant 20$ events $\cdot \mathrm{h}^{-1}$ who did not tolerate nCPAP showed increased adjusted risks of mortality compared with patients with an $\mathrm{AHI}<20$ events $\cdot \mathrm{h}^{-1}$ (hazard ratio 2.69) and to those with $\mathrm{AHI} \geqslant 20$ events $\cdot \mathrm{h}^{-1}$ who tolerated nCPAP (hazard ratio 1.58). However, as recognised by those authors, the open-label, nonrandomised design of their study and the low percentage of adherence to treatment (nearly 30\% of patients) are important limitations that should be taken into account. It is likely that patients who did not tolerate nCPAP were those with more severe disease, greater functional impairment or previous stroke, and poor prognosis. However, nCPAP therapy was introduced in the stable phase of stroke, $\geqslant 2$ months from the onset of symptoms, when the expected functional recovery has already been taken place and data regarding the effect of nCPAP on neurological outcome cannot be assessed. Nevertheless, another important difference between both studies is the length of followup, since the majority of cardiovascular events occurred after 30 months in the referred study, and a follow-up of 24 months in our study might not be prolonged enough (most likely unpowered for secondary outcome of stroke).

However, our results are similar to those reported in a recent study [53] in which recurrence and vascular death at a mean of 12 months (SD 15 days) was $19.8 \%$ in patients with ischaemic stroke with polyvascular atherothrombotic disease compared with $12.4 \%$ in patients with monovascular atherothrombotic disease. In our study, the rate of cardiovascular events (including cardiac ischaemic events, stroke recurrence and 
cardiovascular death) was $12 \%$ (15 out of 126 subjects; $12.3 \%$ in the nCPAP group and $11.6 \%$ in the control group) at $2 \mathrm{yrs}$, but almost all events occurred within the first year. However, the mortality in our series was generally low because more severe stroke patients were excluded. In this respect, the percentage of patients with minimal or negligible neurological dysfunction was high (close to $30 \%$ in some scales). All of them seemed to be explained in part by the selection related to the inclusion criteria: age $<75$ yrs, first episode of stroke and ischaemic stroke, and consciousness to cooperate. Therefore, the nonsevere characteristics of stroke patients and the limited followup period may account for the low morbidity and mortality observed in the present series. Moreover, the lack of statistical power may be a matter of concern, although differences in mortality between the nCPAP and control groups would be achieved if 100 patients had been included in each group.

Positive effects of nCPAP in the long term cannot be excluded. Moreover, early nCPAP treatment in ischaemic stroke patients may be beneficial to prevent cardiovascular events on a longterm basis. In the present series of patients, nCPAP was started after a mean $\pm S D$ of $4.6 \pm 2.8$ days after the onset of stroke following completion of diagnostic studies. Whether a much earlier nCPAP approach may have lead to a more positive results in favour of the nCPAP group is unknown.

In a pilot study of 12 stroke patients who, within $48 \mathrm{~h}$ of acute stroke onset, underwent sleep studies, nocturnal noninvasive blood pressure studies during CPAP and spontaneous breathing, and cerebral blood flow velocity measurement in the middle cerebral artery with transcranial Doppler during spontaneous breathing and CPAP, possible harmful haemodynamic effects of CPAP were reported at higher pressures [54]. However, in our study, neither systemic haemodynamic values nor cerebral circulatory data were recorded.

The degree of hypoxaemia was greater in the nCPAP group than in controls, which, in fact, might potentiate the positive effect of nCPAP despite acting upon a subset of hypothetically more severe patients. However, in a previous study [10], CT90 was not selected as independent predictor of mortality in the logistic regression analysis in stroke patients with sleepdisordered breathing. A higher CT90 in the nCPAP group may be related to a greater BMI and a higher percentage of alcohol abuse in this group.

In summary, early use of nCPAP in first-ever ischaemic stroke patients followed for 24 months seems to accelerate neurological recovery and to delay the appearance of cardiovascular events, although an improvement in survival or in the quality of life of the patients was not shown.

\section{SUPPORT STATEMENT}

Supported in part by grants from FIS (PI08/1514, PI05/0678, PI05/2631 and PI05/0772), Madrid, Spain.

\section{CLINICAL TRIAL}

This study is registered at ClinicalTrials.gov with identifier number NCT00202501.

\section{STATEMENT OF INTEREST}

None declared.

\section{ACKNOWLEDGEMENTS}

The authors affiliations are: O. Parra, Servicio de Neumología, Hospital Universitari del Sagrat Cor, Universitat de Barcelona, Barcelona, and CIBER Enfermedades Respiratorias (CiberRes CB06/06), Ministerio e Cinecia e Investigación, Madrid, Spain; Á. Sánchez-Armengol, Unidad de Trastornos Respiratorios del Sueño, Unidad Médico Quirúrgica de Enfermedades Respiratorias, Barcelona, Spain; M. Bonnin, Servicio de Neumología, Hospital Universitari del Sagrat Cor, Universitat de Barcelona, Barcelona, Spain; A. Arboix, CIBER Enfermedades Respiratorias (CiberRes CB06/06), Ministerio e Cinecia e Investigación, Madrid, and Enfermedades Cerebrovasculares, Servicio de Neurología, Hospital Universitari del Sagrat Cor, Universitat de Barcelona, Barcelona, Spain; F. Campos-Rodríguez, Servicio de Neumología, Hospital Universitario de Valme, Seville, Spain; J. Pérez-Ronchel, Servicio de Neumología, Hospital Universitario de Valme, Seville, Spain; J. DuránCantolla, CIBER Enfermedades Respiratorias (CiberRes CB06/06), Ministerio e Cinecia e Investigación, Madrid, and Unidad de Sueño, Departamento de Medicina, Hospital Txagorritxu, Universidad del País Vasco, Vitoria-Gasteiz, Spain; G. de la Torre, Unidad de Sueño, Departamento de Medicina, Hospital Txagorritxu, Universidad del País Vasco, Vitoria-Gasteiz, Spain; J.R. González Marcos, Servicio de Neurología, Hospitales Universitarios Virgen del Rocío, Seville, Spain; M. de la Peña, Unidad de Sueño, Servicio de Neumología, Hospital Universitario Son Dureta, Palma de Mallorca, Spain; M. Carmen Jiménez, Servicio de Neurología, Hospital Universitario Son Dureta, Palma de Mallorca, Spain; F. Masa, Servicio de Neumología, Hospital San Pedro de Alcántara, Cáceres and CIBER Enfermedades Respiratorias (CiberRes CB06/06), Ministerio e Cinecia e Investigación, Madrid, Spain; I. Casado, Servicio de Neurología, Hospital San Pedro de Alcántara, Cáceres, Spain; M. Luz Alonso, CIBER Enfermedades Respiratorias (CiberRes CB06/06), Ministerio e Cinecia e Investigación, Madrid, and Unidad de Sueño, Sección de Neumología, Burgos, Spain; and J.L. Macarrón, Sección de Neurología, Hospital General Yagüe, Burgos, Spain.

We thank M. Pulido (Consulting Service on Biomedical Publishing, Institut Municipal d'Investigació Mèdica, Barcelona, Spain) for editing the manuscript and editorial assistance, and Clever Instruments SL (Barcelona) for statistical analysis.

\section{REFERENCES}

1 Lavie P, Herer P, Peled R, et al. Mortality in sleep apnea patients: a multivariate analysis of risk factors. Sleep 1995; 18: 149-157.

2 Marin JM, Carrizo SJ, Vicente E, et al. Long-term cardiovascular outcomes in men with obstructive sleep apnoea-hypopnoea with or without treatment with continuous positive airway pressure: an observational study. Lancet 2005; 365: 1046-1053.

3 Young T, Finn L, Peppard PE, et al. Sleep disordered breathing and mortality: eighteen-year follow-up of the Wisconsin sleep cohort. Sleep 2008; 31: 1071-1078.

4 Bassetti C, Aldrich MS, Quint D. Sleep-disordered breathing in patients with acute supra- and infratentorial strokes. A prospective study of 39 patients. Stroke 1997; 28: 1765-1772.

5 Arzt M, Young T, Finn L, et al. Association of sleep-disordered breathing and the occurrence of stroke. Am J Respir Crit Care Med 2005; 172: 1447-1451.

6 Parra O, Arboix A, Bechich S, et al. Time course of sleep-related breathing disorders in first-ever stroke or transient ischemic attack. Am J Respir Crit Care Med 2000; 161: 375-380.

7 Shahar E, Whitney CW, Redline S, et al. Sleep-disordered breathing and cardiovascular disease: cross-sectional results of the Sleep Heart Health Study. Am J Respir Crit Care Med 2001; 163: 19-25.

8 Yaggi HK, Concato J, Kernan WN, et al. Obstructive sleep apnea as a risk factor for stroke and death. N Engl J Med 2005; 353: 2034-2041.

9 Good DC, Henkle JQ, Gelber D, et al. Sleep-disordered breathing and poor functional outcome after stroke. Stroke 1996; 27: 252-259. 
10 Parra O, Arboix A, Montserrat JM, et al. Sleep-related breathing disorders: impact on mortality of cerebrovascular disease. Eur Respir J 2004; 24: 267-272.

11 Sahlin C, Sandberg O, Gustafson Y, et al. Obstructive sleep apnea is a risk factor for death in patients with stroke: a 10-year followup. Arch Intern Med 2008; 168: 297-301.

12 Bålfors EM, Franklin KA. Impairment of cerebral perfusion during obstructive sleep apneas. Am J Respir Crit Care Med 1994; 150: 1587-1591.

13 Drager LF, Bortolotto LA, Lorenzi MC, et al. Early signs of atherosclerosis in obstructive sleep apnea. Am J Respir Crit Care Med 2005; 172: 613-618.

14 Schulz R, Hummel C, Heinemann S, et al. Serum levels of vascular endothelial growth factor are elevated in patients with obstructive sleep apnea and severe nighttime hypoxia. Am J Respir Crit Care Med 2002; 165: 67-70.

15 Minoguchi K, Yokoe T, Tazaki T, et al. Increased carotid intimamedia thickness and serum inflammatory markers in obstructive sleep apnea. Am J Respir Crit Care Med 2005; 172: 625-630.

16 Sullivan CE, Issa FG, Berthon-Jones $\mathrm{M}$, et al. Reversal of obstructive sleep apnoea by continuous positive airway pressure applied through the nares. Lancet 1981; 1: 862-865.

17 Montserrat JM, Ferrer M, Hernandez L, et al. Effectiveness of CPAP treatment in daytime function in sleep apnea syndrome: a randomized controlled study with an optimized placebo. Am J Respir Crit Care Med 2001; 164: 608-613.

18 Faccenda JF, Mackay TW, Boon NA, et al. Randomized placebo controlled trial of continuous positive airway pressure on blood pressure in the sleep apnea-hypopnea syndrome. Am J Respir Crit Care Med 2001; 163: 344-348.

19 Pepperell JC, Ramdassingh-Dow S, Crosthwaite N, et al. Ambulatory blood pressure after therapeutic and subtherapeutic nasal continuous positive airway pressure for obstructive sleep apnoea: a randomised parallel trial. Lancet 2002; 359: 204-210.

20 Becker HF, Jerrentrup A, Ploch T, et al. Effect of nasal continuous positive airway pressure treatment on blood pressure in patients with obstructive sleep apnea. Circulation 2003; 107: 68-73.

21 Campos-Rodriguez F, Grilo-Reina A, Perez-Ronchel J, et al. Effect of continuous positive airway pressure on ambulatory BP in patients with sleep apnea and hypertension: a placebo-controlled trial. Chest 2006; 129: 1459-1467.

22 Lattimore JL, Wilcox I, Skilton M, et al. Treatment of obstructive sleep apnoea leads to improved microvascular endothelial function in the systemic circulation. Thorax 2006; 61: 491-495.

23 Drager LF, Bortolotto LA, Figueiredo AC, et al. Effects of continuous positive airway pressure on early signs of atherosclerosis in obstructive sleep apnea. Am J Respir Crit Care Med 2007; 176: 706-712.

24 Minoguchi K, Yokoe T, Tazaki T, et al. Silent brain infarction and platelet activation in obstructive sleep apnea. Am J Respir Crit Care Med 2007; 175: 612-617.

25 Muñoz R, Doran-Cantilla J, Martínez-Vila E, et al. Severe sleep apnea and risk of ischemic stroke in the elderly stroke. Stroke 2006; 37: 2317-2321.

26 Wessendorf TE, Wang Y-M, Thilmann AF, et al. Treatment of obstructive sleep apnoea with nasal continuous positive airway pressure in stroke. Eur Respir J 2001; 18: 623-629.

27 Sandberg O, Franklin KA, Bucht G, et al. Nasal continuous positive airway pressure in stroke patients with sleep apnoea: a randomized treatment study. Eur Respir J 2001; 18: 630-634.

28 Parra O. Sleep-disordered breathing and stroke: is there a rationale for treatment? Eur Respir J 2001; 18: 619-622.

29 Bassetti CL, Milanova M, Gugger M. Sleep-disordered breathing and acute ischemic stroke: diagnosis, risk factors, treatment, evolution, and long-term clinical outcome. Stroke 2006; 37: 967-972.

30 Harbison J, Ford GA, Gibson GJ. Nasal continuous positive airway pressure for sleep apnoea following stroke. Eur Respir J 2002; 19: 1216-1217.
31 Hui DS, Choy DK, Wong LK, et al. Prevalence of sleep-disordered breathing and continuous positive airway pressure compliance: results in chinese patients with first-ever ischemic stroke. Chest 2002; 122: 852-860.

32 Palombini L, Guilleminault C. Stroke and treatment with nasal CPAP. Eur J Neurol 2006; 13: 198-200.

33 Hsu CY, Vennelle M, Li HY, et al. Sleep-disordered breathing after stroke: a randomised controlled trial of continuous positive airway pressure. J Neurol Neurosurg Psychiatry 2006; 77: 1143-1149.

34 Martinez-Garcia MA, Soler-Cataluña JJ, Ejarque-Martinez L, et al. CPAP treatment reduces mortality in ischemic stroke patients with obstructive sleep apnea. Am J Respir Crit Care Med 2009; 180: 36-41.

35 Arboix A, Morcillo C, García-Erols L, et al. Different vascular risk factor profiles in ischemic stroke subtypes: a study from the "Sagrat Cor Hospital of Barcelona Stroke Registry". Acta Neurol Scand 2000; 102: 264-270.

36 Arboix A. Alvarez-Sabín J, Soler L, for the Cerebrovascular Study Group of the Spanish Society of Neurology. Nomenclatura de las enfermedades vasculares cerebrales. Neurologia 1998; 13: Suppl. 1, 1-10.

37 Mahoney FI, Barthel DW. Functional evaluation: the Barthel Index. Md State Med 1965; 14: 61-65.

38 Côté R, Battista RN, Shurvell BL, et al. The Canadian Neurological Scale: a preliminary study in acute stroke. Stroke 1986; 17: 731-737.

39 Van Swieten JC, Koudstaal PJ, Visser MC, et al. Interobserver agreement for the assessment of handicap in stroke patients. Stroke 1988; 19: 604-607.

40 Jenkinson C, Davies R, Mullins R, et al. Randomised prospective parallel trial of therapeutic nasal continuous positive airway pressure (NCPAP) against sub- therapeutic NCPAP for obstructive sleep apnoea. Lancet 1999; 353: 2100-2105.

41 Johns MW. A new method for measuring daytime sleepiness: the Epworth Sleepiness Scale. Sleep 1991; 14: 540-545.

42 Molina M, Hernández L, Duran J, et al. Protocol to evaluate automatic continuous positive airway pressure. Assessment of the usefulness of the Autoset-T device to determine optimal pressure for treating sleep apnea/hypopnea syndrome. Arch Bronconeumol 2003; 39: 118-125.

43 Masa JF, Jiménez A, Durán J, et al. Alternative methods of titrating continuous positive airway pressure: a large multicenter study. Am J Respir Crit Care Med 2004; 170: 1218-24.

44 Ancoli-Israel S, Kripke DF, Klauber MR, et al. Sleep disordered breathing in community-dwelling elderly. Sleep 1991; 14: 486-495.

45 Calvin AD, Somers VK. Obstructive sleep apnea and risk of stroke: time for a trial. Nat Clin Pract Cardiovasc Med 2009; 6: 90-91.

46 Alarcón A, León C, Maimó A, et al. Compliance with nasal continuous positive airway pressure (CPAP) treatment in sleep apnea-hypopnea syndrome. Arch Bronconeumol 1995; 31: 56-61.

47 Iranzo A, Santamaría J, Berenguer J, et al. Prevalence and clinical importance of sleep apnea in the first night after cerebral infarction. Neurology 2002; 58: 911-916.

48 Duncan PW, Wallace D, Lai SM, et al. The stroke impact scale version 2.0. Evaluation of reliability, validity, and sensitivity to change. Stroke 199, 30: 2131-2140.

49 Nishibayashi M, Miyamoto M, Miyamoto T, et al. Correlation between severity of obstructive sleep apnea and prevalence of silent cerebrovascular lesions. J Clin Sleep Med 2008; 15: 242-247.

50 Redline S, Strauss ME, Adams N, et al. Neuropsychological function in mild sleep-disordered breathing. Sleep 1997; 20: 160-167.

51 Barnes M, McEvoy RD, Banks S, et al. Efficacy of positive airway pressure and oral appliance in mild to moderate obstructive sleep apnea. Am J Respir Crit Care Med 2004; 170: 656-664.

52 Morrell MJ, McRobbie DW, Quest RA, et al. Changes in brain morphology associated with obstructive sleep apnea. Sleep Med 2003; 4: 451-454.

53 Blanco M, Sobrino T, Montaner J, et al. Stroke with polyvascular atherothrombotic disease. Atherosclerosis 2010; 208: 587-592.

54 Scala R, Turkington PM, Wanklyn P, et al. Acceptance, effectiveness and safety of continuous positive airway pressure in acute stroke: a pilot study. Respir Med 2009; 103: 59-66. 